\title{
Exposé Sommaire des Etudes Relatives à l'Aimantation de Matériaux Volcaniques
}

\author{
Par A. ROCHE \\ Institut et Observatoire de Physique du Globe du Puy de Dôme, \\ Université de Clermont
}

\section{Résumé}

L'auteur rappelle très brièvement les résultats qu'il a obtenus au cours de recherches effectuées de 1947 à 1953 sur les roches volcaniques d'Auvergne et du Velay.

Les inversions d'aimantation constatées paraissent caractériser tous les matériaux volcaniques de certains âges quelle que soit leur nature pétrographique.

Ces observations semblent favorables à l'hypothèse d'inversions réelles du champ magnétique terrestre qui se seraient répétées plusieurs fois durant L'Ere Tertiaire.

Les roches étudiées jusqu'à présent appartiennent toutes au Massif Central de la France (Auvergne et Velay). L'époque de leur mise en place varie depuis le Tertiaire moyen (Stampien) jusqu'au Quaternaire récent. Elles appartiennent à des types pétrographiques variés (trachytes, andésites, téphrites, basaltes). Des specimens d'argiles ou de marnes pris au voisinage de masses volcaniques ont été aussi étudiés.

Les principaux résultats obtenus sont les suivants:

1/ A l'exception d'anomalies très localisées, une même unité volcanique: coulée, dyke ou neck, est caractérisée par une direction et un sens d'aimantation qui lui sont propres.

2/ Les directions d'aimantation observées s'écartent relativement peu de la direction actuelle du champ magnétique terrestre au lieu considéré.

3/ Le sens d'aimantation est soit le sens du champ terrestre actuel, soit le sens inverse.

4/ Les roches du Quaternaire récent ont un sens d'aimantation en accord avec celui du champ terrestre actuel (sens normal).

5/ Les roches plus anciennes ont une aimantation, soit de sens normal, soit de sens inverse.

6/ Le groupement des aimantations normales et inverses parait se faire en fonction de l'âge des roches étudiées.

J'ai observé des aimantations de sens inverse pour les périodes suivantes:

Pléistocène ancien et Pliocène récent (Villafranchien). Limite du Pliocène 
inférieur et du Miocène supérieur (Astien-Pontien), Oligocène (Stampien supérieurAquitanien).

7/ Les argiles et marnes récoltées au voisinage immédiat de roches volcaniques à aimantation inversée ont toujours présenté aussi une aimantation inversée bien que leurs constituants minéralogiques soient d'origine indépendante.

Interprétation des faits observés :

2 hypothèses sont en présence:

l'une attribuant les inversions d'aimantation à des inversions réelles du champ magnétique terrestre, l'autre attribuant les inversions au jeu de mécanismes internes à la roche, le champ magnétique terrestre ayant gardé un sens constant. La valabilité de cette $2^{\text {e }}$ hypothèse a été confirmée à la suite des études théoriques de L. Néel et des résultats expérimentaux obtenus par T. Nagata. Les nombreux travaux de laboratoire effectués (J. Graham, J. Hospers, T. Nagata, J. Roquet, E. Thellier ect) ont montré des aspects nouveaux et intéressants de la question, mais n'ont pas apporté d'argument décisif en faveur de l'une ou de l'autre hypothèse. On peut retenir comme arguments favorables à l'hypothèse de changements de sens effectifs du champ magnétique terrestre le fait que les aimantations se groupent selon l'âge des roches et le fait que les argiles et marnes récoltées au voisinage de roches volcaniques présentent le même sens d'aimantation qu'elles.

Suggestions proposées pour les recherches ultérieures sur le terrain.

1/ Récolter chaque fois que cela est possible des roches sédimentaires au voisinage immédiat de coulées ou de dykes et vérifier le sens de leur aimantation comparativement à celui des roches volcaniques.

2/ S’attacher à récolter des échantillons volcaniques bien datés. Les méthodes radioactives donnent une précision relative faible pour le tertiaire récent et le quaternaire. On pourrait utiliser la méthode stratigraphique en examinant de préférence des coulées encadrées par des couches sédimentaires fossilifères.

Les intervalles de temps pendant lesquels les aimantations paraissent garder un sens constant sont brefs par rapport aux durées des périodes géologiques et l'on ne peut guère espérer établir une corrélation chronologique entre les résultats obtenus en différentes régions du globe pour des roches assez anciennes.

Par contre il serait intéressant de préciser la date de la mise en place des roches à aimantation inversée du Pléistocène ancien et du Villafranchien. Si l'on parvenait à établir pour cette période des dates limites entre lesquelles toutes les roches observables présentent l'aimantation inversée et à l'extérieur desquelles elles présentent l'aimantation normale, on aurait là un argument très fort en faveur de changements de sens réels du champ terrestre et un espoir de rattacher ces changements à un caractère astronomique ou géologique de l'histoire du globe. 


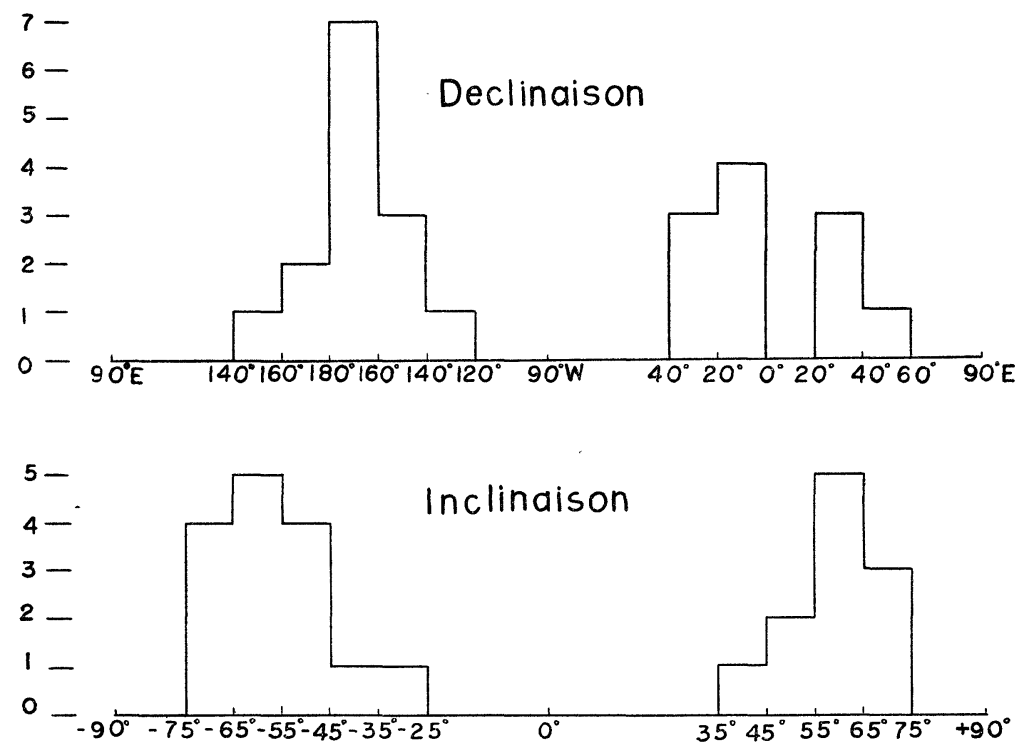

Fig. 1 Diagramme de la fréquence des unités volcaniques en fonction de la direction de leur aimantation thermorémanente. 